\title{
Research on Bio-amplifier for ECG Recording
}

\author{
Qingjun $\mathrm{Zhu}^{1 *}$
}

\author{
${ }^{I}$ Rose-Hulman Institute of Technology, 5500 Wabash AVE, Terre Haute, IN, USA, 47803 \\ *Corresponding author's e-mail: Zhuq1@rose-hulman.edu
}

\begin{abstract}
The emergence and functions of biological amplifiers not only enrich the medical equipment market, but more importantly, it provides more treatment opportunities for patients and medical staff. Biological amplifiers have been widely used in electrocardiogram (ECG) medical equipment. Doctors have obtained more comprehensive, detailed, and accurate data and diagrams with the enhanced functions of ECG to facilitate more feasible and safer treatment plans. Therefore, through the literature review method, this paper studies the enhanced functions of bio-amplifier on ECG. In the study of two ECG Bio-amplifiers, the paper first finds that the use of bio-amplifier on ECG plays a function in improving the accuracy of cardiac indicators. Secondly, this paper finds that ECG Isolation Amplifier improves the stability of ECG by limiting the current, so its safety is guaranteed. For doctors who use defibrillation to save patients' lives, ECG equipment improves the benefits of saving heart disease patients through high reliability. Moreover, ECG Instrumentation Bio-amplifier resists electromagnetic effects from other electronic devices by high common-mode rejection, which reduces doctors' concerns about data errors.
\end{abstract}

Keywords: Medical Equipment, ECG, bio-amplifiers, Isolation \& Instrumentation Amplifier

\section{INTRODUCTION}

In medical equipment, bio-amplifiers, which are closely related to ECG functions and characteristics, have always played an important role. Therefore, it is significant to study the different needs of ECG-based bio-amplifiers in specific medical situations aiming at the requirements of different medical conditions for different medical equipment. This research is dedicated to describing two different types of bio-amplifiers based on ECG and observing their functions in different medical places, which will provide a further theoretical basis for future research on the requirements of different medical equipment in various medical conditions.

\section{INTRODUCTION TO BIO-AMPLIFIER}

Before studying the Bio-amplifiers based on ECG, it is necessary to define and describe ECG. ECG is the abbreviation of electrocardiogram, it is a kind of biomedical equipment used to display clinical patients' heartbeat speed, rhythm, and the time and intensity of electrical impulses moving within the heart. Therefore, the main function of ECG is to present the biological indicators related to a patient's heart. Usually, ECG is displayed through a microcomputer device. As shown in
Figure 1 below, ECG is a program that analyzes the heart of a patient.

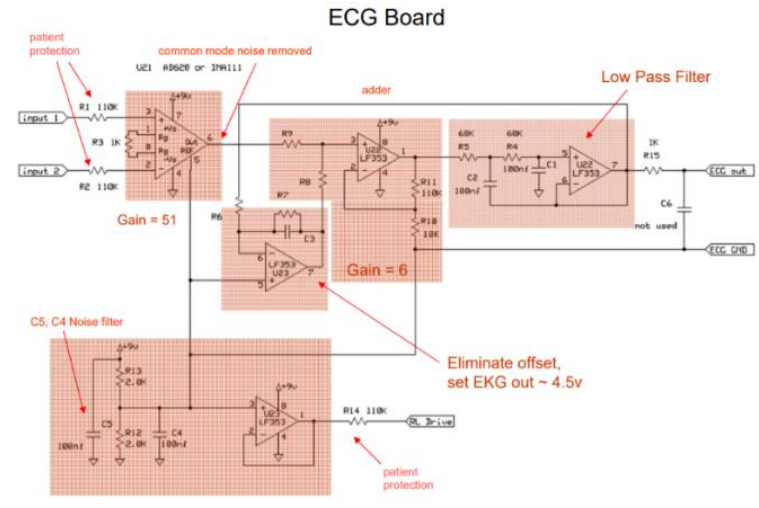

Figure 1 Electrocardiogram (ECG)

As the control program is displayed on the microcomputer, a patient's heartbeat and other indicators are presented. An operator can display physiological indicators such as heartbeat by operating the keyboard on the microcomputer. Therefore, the microcomputer plays an indispensable role in displaying ECG. By operating the microcomputer, the lead selector determines which electrodes are required for a specific lead, and the operator automatically controls the microcomputer to connect them to the rest of the circuit[1]. Therefore, the 
microcomputer is mainly responsible for the ECG display of cardiac indicators. In biomedicine, ECG monitors the patient's heart while displaying the patient's heart indicators. Therefore, another medical instrument, Cardiac monitors, is closely related to ECG. As it is argued, "Clinical applications of continuous monitoring of the ECG and heart rate are made possible by cardiac monitor"[2]. With the support of these medical devices, ECG can quickly and accurately display the physiological indicators of the heart of a patient. Therefore, one of the advantages of ECG is that it can help a doctor and a patient quickly display abnormalities about a patient's heart. With the support of sophisticated instruments such as the microcomputer and cardiac monitor, abnormal indicators related to the heart can be quickly displayed. It not only achieved rapid and effective success in the development of biomedicine, but it also helped doctors shorten the time in diagnosis and treatment.

Bio-amplifier is an amplifier applied to biomedicine. Generally, the amplitude and frequency of biological electronic signals in the human body are low, so a single device such as a microcomputer and a heart detector cannot accurately capture the signal. Therefore, "the outputs from these amplifiers are used for further analysis and they appear as ECG, EMG, or any bioelectric waveforms" [3]. Bio-amplifier is a signal amplifier used to enhance electronic signal reception and analysis [10]. One type of Bio-amplifier is the differential amplifier.

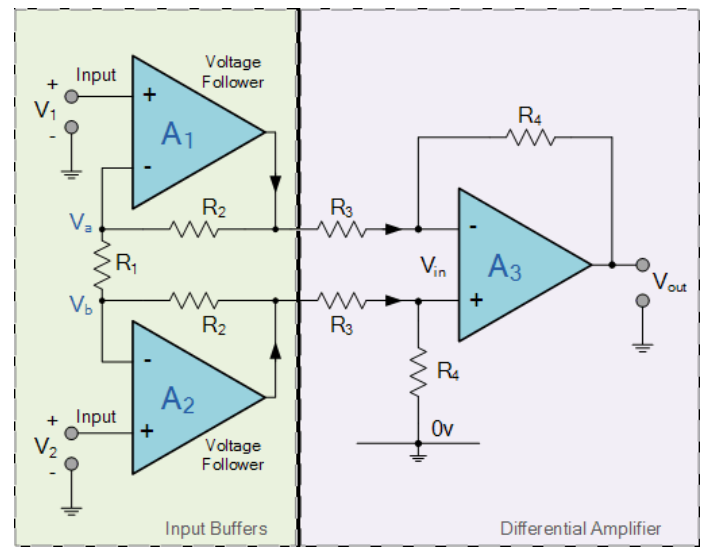

Figure 2- Differential amplifier
This type of amplifier is generally used in input-stage medical amplifiers. When measuring the voltage difference between two bioelectronics, a differential amplifier is used (See Figure 2 below). It is usually applied to collect the voltage from the arm to the leg and the heart, and the voltage from the scalp to the brain. The differential amplifier can resist common-mode interference signals, and it has good stability and versatility[3]. The second type is the operational amplifier, which is a high gain differential amplifier (See Figure 3 below). An operational amplifier is usually unstable and difficult to control due to its high gain, and it includes inverting amplifier and a non-inverting amplifier. The third type is instrumentation amplifier. This amplifier is similar to the differential amplifier, but it is a differential amplifier with an additional input buffer stage. This makes it easy to match with the preceding stage[3]. In addition to similar high gain characteristics, the instrumentation amplifier also has high input resistance and low bias voltage characteristics. Another type of amplifier is the isolation amplifier (See Figure 4 below). When using current-driven medical equipment, safety is the most important for patients. "Isolation amplifiers can be used to break ground loops, eliminate source ground connections, and provide isolation protection to patient and electronic equipment"[3]. It can be found that the main function of the Isolation amplifier is to protect the patient from all possible hazards of electric shock. Besides, another function of the Isolation amplifier is that it can remove noise.

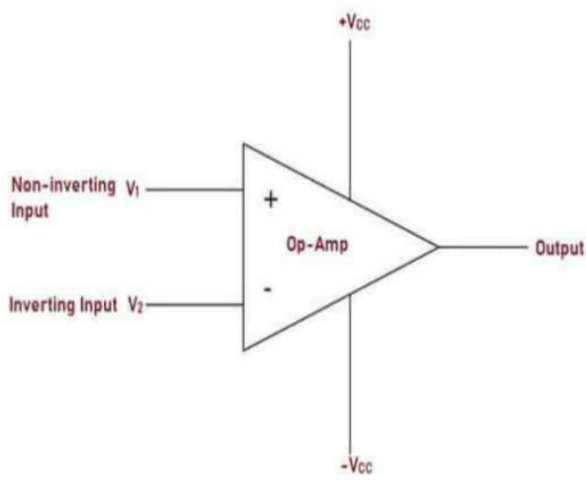

Figure 3- Operational amplifier 


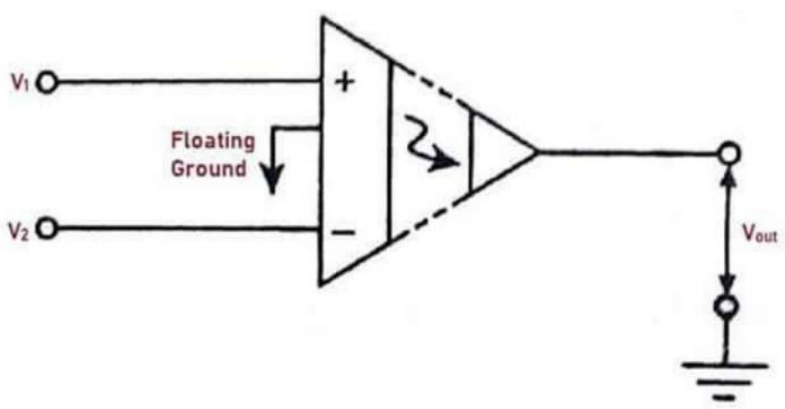

Figure 4- Isolation amplifier

\section{BIO-AMPLIFIER IN ECG}

In the use of ECG, Bio-amplifier is indispensable because it provides a higher level of biological electronic signal detection and reception. As discussed earlier, Bioamplifier is a signal enhancer. After being applied to the ECG, the physiological indicators related to the patient's heart can be quickly and accurately displayed by the microcomputer. Therefore, the processing steps of ECG are very critical, especially when it involves choosing which type of Bio-amplifier.

In the signal processing of ECG, the first step is to amplify the signals of the patient's heart. These signals are biological electronic signals of the human body to display indicators of the heart. For example, the heartbeat speed, rhyme, frequency, and other signals need to be displayed on the microcomputer through the Bioamplifier. However, since the bioelectronic signals that originate from the human skin are also detected by ECG at the same time after Bio-amplifier amplifies the biological electronic signals, the interference noise needs to be removed so that the captured signals are from the heart area. Normally, the noise removed is above $23 \mathrm{~Hz}$ to get the frequencies near the heart [4]. The third step is to apply bias for single-supply operation and enhance common-mode rejection. The main purpose of this step is to resist common-mode interference signals. The function of removing interferences of the bio-amplifier is demonstrated in this step. The last step is to compensate the DC offset. As mentioned earlier, the bio-amplifier is driven by an electric current, so the electric current may cause an electric shock to the equipment and the patient. Therefore, this step is to offset the damage of biological electrons to ECG.

It can be found from the signal processing of ECG that it also determines the choice of bio-amplifier. Although the functions of bio-amplifiers are diverse, in the use of ECG, a special function is to remove noise. By reviewing the discussion of different types of bioamplifiers, it can be found that the isolation bio-amplifier has the function of removing noise[5]. Secondly, to make ECG signal processing have higher common-mode rejection and input impedance, another bio-amplifier that ECG needs is an instrumentation amplifier.

\section{FIRST TYPE BIO-AMPLIFIER: ECG ISOLATION AMPLIFIER}

Isolation amplifiers are usually applied in ECG to capture signals. In this process, the signals generated by leads are sent to the electrosurgery filter, which can reduce the interference between electrosurgery and radiofrequency. This is a process of isolation amplifiers to remove noise and reduce interference signals. Secondly, the isolation amplifier can withstand excessive voltage and protect the voltage during defibrillation. In this process, due to the high impedance of the isolation amplifier, it can reduce the current by lowering the voltage. This is also the main reason for the high stability of the isolation amplifier. As demonstrated in the description of the isolation amplifier, one of its functions is to protect patients and devices from the hazards of electric shocks. As the isolation amplifier goes to the lead selector switch, it selects the required configuration and then the lead selection output enters the DC amplifier[3]. Subsequently, after the adjustment of the transformer, the current decreases and remains stable. The ECG signal is output by the synchronous modulator and displayed by the microcomputer.

During the process of the isolation amplifier, it can be found that it has several main advantages in ECG. Firstly, the isolation amplifier removes ripple noise. For ECG, this will help reduce the interference of noise on heartrelated indicators. Secondly, the isolation amplifier is also pretty. As it is pointed out, "The isolation stage serves the galvanic decoupling of the patient from the measuring equipment and provides safety from electrical hazard"[6]. Third, the isolation amplifier has a strong anti-magnetic ability[7]. In other words, in ECG, due to the isolation amplifier, it is hardly affected by mobile phones, computers, or other magnetic objects. Finally, due to the high impedance of the isolation amplifier and the reduction of the current to the transformer, the isolation amplifier has high gain stability. 


\section{SECOND TYPE BIO-AMPLIFIER: ECG INSTRUMENTATION BIO-AMPLIFIER}

Since the Instrumentation bio-amplifier has lower signal noise, it is often applied to ECG. As it is argued, "As the technology of biomedical instrumentation amplifier has moved towards portability, lower power consumption is highly desirable" [8]. The instrumentation biological amplifier has an input buffer, which makes the instrumentation biological amplifier easy to match with the preceding stage. Therefore, the instrumentation biological amplifier is easier to work in ECG. The low DC bias means that the ECG is hardly affected by other change points. In addition, a characteristic of the Instrumentation bio-amplifier is the high common-mode rejection ratio [9]. High commonmode rejection means higher resistance to stronger current interference. Instrumentation bio-amplifier is a very precise medical instrument, so it must keep the interferences as low as possible when detecting ECG signals.

Therefore, one of the advantages of the Instrumentation biological amplifier is that it requires a low power supply, and it can improve the accuracy of physiological indicators, the heart-related indicators in ECG. Second, it is monitored to understand heart health [10]. Third, like isolation amplifiers, Instrumentation biological amplifiers have very low noise. Finally, judging from the working principle of the Instrumentation biological amplifier in ECG, one of its most prominent advantages is that it is easy to match with preceding stages.

\section{CONCLUSION}

Since ECG can play a vital role in quickly displaying the patient's cardiac indicators, medical staff have an urgent demand for ECG, especially when bio-amplifiers enhance the function of ECG in medical equipment. Whether it is a differential amplifier, an operational amplifier, or an isolation amplifier, they all provide medical equipment with more versatile and powerful functions by their unique functions, so they all play a vital role in biological amplifiers. From resisting common-mode interference signals to high gain, to improving safety performance, these amplifiers are regarded as treasures by the medical equipment market, especially in ECG applications. Through the study of the ECG isolation amplifier, it can be found that it not only reduces the signal interference generated by electronic equipment to ECG but also protects the voltage during defibrillation, which avoids accidents that may be caused to patients due to excessive voltage. For ECG Instrumentation Bio-amplifier that accurately detects cardiac indicators of patients, it provides doctors with more accurate data. With the advantages of noise reduction and portability, it is widely accepted in ECG medical equipment.

\section{REFERENCES}

[1] Akdogan, Kurtulus Erinc. "Biopotential Amplifiers." pp. 1-94.

https://mece493.cankaya.edu.tr/uploads/files/Biopo tential\%20Amplifiers.pdf

[2] Shamekhi. "Bioinstrument: Lecture 4 Biopotential Amplifiers." Sahand University of Technology, Summer 2016, pp. 1-26 http://fa.bme.sut.ac.ir/Downloads/AcademicStaff/3 /Courses/4/Bioinstrument\%204\%20(BioAmplifiers )$\cdot p d f$

[3] "Basics of Biomedical Instrumentation." pp. 1-20. https://rmd.ac.in/dept/ece/Supporting_Online_\%20 Materials/5/BBI/unit3.pdf

[4] “Amplifiers: Class A, B, AB, D.” 2020, pp. 1-59. https://web.mit.edu/6.101/www/s2020/handouts/L 08.pdf

[5] "Wide bandwidth BioAmplifier." http://www.psylab.com/html/default_bioamp.htm

[6] Nagel, Joachim H. "70 Biopotential Amplifiers." CRC Press LLC, 2000, pp. 1-15. http://fisica.uc.pt/data/20062007/apontamentos/apn t_134_6.pdf

[7] Sommerville, Tom. "Isolation Amps Hike Accuracy and Reliability Isolation Amplifiers Protect Critical Circuitry From Damage and Enhance Performance." Burr-Brown Corporation, 1994, pp. 1-6. https://www.ti.com/lit/an/sboa064/sboa064.pdf?ts= $1631164528550 \&$ ref_url=https $\% 253 \mathrm{~A} \% 252 \mathrm{~F} \% 25$ 2Fwww.google.ca\%252F

[8] Lim, E. C. M., et al. "Design of Low-Power LowVoltage Biomedical Amplifier for Electrocardiogram Signal Recording.” IEEE, 2007, pp. 191-194.

[9] Paulus, Daniel, and Thomas Meier. "ECG-Amplifier." 2009 , pp. $1-12$. http://wwwmayr.in.tum.de/konferenzen/MBJass2009/courses/1/Paulus.pdf

[10] Chakrasthitha. "Biopotential Amplifier - How it Works, Types, Applications \& Advantages." Electricalfundablog, 2021. https://electricalfundablog.com/biopotentialamplifier-works-types-applications/ 